\title{
Comparação entre as Ementas de um Curso de Mecânica Quântica e Física Moderna
}

Comparative study on the contents of Quantum Mechanics and Modern Physics courses

\author{
Weslei Silva de Araújo e Clóves Gonçalves Rodrigues* \\ Núcleo de Pesquisa em Física, Departamento de Matemática e Física \\ Universidade Católica de Goiás, CP 86, 74605-010, Goiânia, Goiás, Brazil
}

Recebido em 19 de Abril 2001. Aceito em 17 de Setembro 2001.

\begin{abstract}
Neste trabalho fazemos uma comparação entre o curso de Física Moderna (ministrado nos cursos de Licenciatura e Bacharelado em Física) e o de Mecânica Quântica ministrado apenas nos cursos de Bacharelado em Física. A comparação entre o conteúdo ministrado no curso de Física Moderna com o ministrado no curso de Mecânica Quântica foi feita a partir dos livros de textos mais adotados no ensino destas duas disciplinas no país. Abordamos também a importância do curso de Física Moderna para os licenciandos em Física.
\end{abstract}

In this work, we make a comparison between the course of Modern Physics (taught on the Physics Teacher Preparation and Bachelor of Physics Programs) and the Quantum Mechanics' course taught only on the Bachelor of Physics Program. The comparison between the contents taught on the Modern Physics course and the contents taught on the Quantum Mechanics' course, was based on the most used books in the teaching of both disciplines in Brazil. We also discuss the importance of the Modern Physics course to future physics teachers. in Physics.

\section{Introdução}

Da concepção da substância primordial da matéria por Tales de Mileto, do atomismo de Demócrito e Leucipo, chegamos, no século XVII, às obras de Galileu Galilei e de Isaac Newton, os quais fundaram a ciência moderna. Ao lado da mecânica, que unificou a descrição dos movimentos no céu e na terra, surgiu no século XIX, a descoberta de James Clerck Maxwell, que unificou a eletricidade, o magnetismo e a óptica.

O começo do século XX marca uma nova etapa na história da Física. De um lado, surge uma nova concepção das trocas de energia entre a matéria e a radiação, contrariando os preconceitos da maioria dos físicos e introduzindo a noção fundamental da estrutura quântica da energia e da matéria.

Com Albert Einstein, eleva-se, a partir de 1905, o belo edifício da teoria da relatividade, que aboliu os preconceitos do espaço absoluto e do tempo absoluto. Ainda a Einstein, devemos a noção de fóton, o quantum de luz que introduziu a dualidade onda-corpúsculo para a radiação e que nos levou à idéia de que as partículas elementares - dada a generalização dessa dualidade à matéria, por Louis de Broglie - não são nem corpúsculos nem ondas, têm propriedades de ambos sendo, portanto, objetos quânticos.
De Einstein, Niels Bohr e de Broglie nasceu a mecânica quântica. Devem ser lembrados também os trabalhos magistrais de Erwin Schrödinger, Werner Heisenberg, Max Born, Pascual Jordan, Paul Adrien Maurice Dirac, Wolfgang Pauli e Arnold Sommerfeld. As discussões de Einstein e Bohr sobre a interação probabilística da mecânica quântica serviram de estímulo ao desenvolvimento desta teoria, a qual nos deu finalmente a compreensão da ligação química, da estabilidade da matéria, do magnetismo e da própria identidade dos átomos.[1]

A partir de 1930, surgiu a física nuclear e dela se destacou, a partir de 1947, a física das partículas elementares.

Dada então a importância da Física Quântica, abordamos neste trabalho duas questões:

i) A importância do curso de Física Moderna para os cursos de Licenciatura em Física;

ii) Uma comparação entre o curso de "Física Moderna" (dado nos cursos de Licenciatura e Bacharelado em Física) e o de "Mecânica Quântica" ministrado apenas nos cursos de Bacharelado em Física.

A comparação entre o conteúdo ministrado no curso de "Física Moderna" com o ministrado no curso de "Mecânica Quântica" foi feita a partir dos livros de

\footnotetext{
*Email: cloves@ucg.br
} 
textos mais adotados no ensino destas duas disciplinas no país: Física Quântica (Gasiorowicz) [2] e Física Quântica (Eisberg e Resnick)[3], adotados, respectivamente, nos cursos de Mecânica Quântica e Física Moderna. O curso de Física Moderna é conhecido também pelo nome "Estrutura da Matéria" ou "Física Atômica e Nuclear" em algumas universidades.

\section{Física Moderna e Mecânica Quântica}

Fazemos nesta seção uma comparação entre as disciplinas Física Moderna e Mecânica Quântica. A disciplina Física Moderna faz parte da grade curricular dos cursos de Licenciatura e Bacharelado em Física. Entretanto, a disciplina Mecânica Quântica faz parte apenas da grade curricular do curso de Bacharelado em Física.

No curso de Física Moderna o primeiro assunto tratado é a radiação térmica, mais precisamente a radiação de corpo negro, sendo feita uma abordagem clássica do assunto, ou seja, analisa-se qual é a visão da física clássica da radiação de corpo negro. Depois dessa análise verifica-se em quais aspectos falha a física clássica. Depois desta análise clássica inicia-se a primeira introdução dos conceitos da chamada "antiga teoria quântica" com a teoria de Planck da radiação de cavidade, que contribuiu para a formulação do postulado de Planck. Com este postulado surgiram implicações que deram origem à antiga teoria quântica. Observamos que nesta introdução a preocupação básica é com a compreensão da teoria quântica.

No curso de Mecânica Quântica verificamos que inicialmente estuda-se a radiação de corpo negro, partindo da visão da Física Clássica, determinando ao mesmo tempo os pontos onde falha a Física Clássica. Depois desse estudo chega-se rapidamente ao postulado de Planck, o mesmo sendo feito para as implicações do postulado. Neste caso o que se faz é um pequeno comentário sobre o assunto, o tema é desenvolvido de forma que o estudante para compreendê-lo precisa antes fazer um estudo mais detalhado, que possibilite ao mesmo uma compreensão efetiva do assunto, do contrário o estudante não conseguirá fazer as ligações necessárias para iniciar o estudo no curso de Mecânica Quântica.

Ao estudarmos o efeito fotoelétrico no curso de Física Moderna notamos que primeiro é feito um estudo histórico do efeito, descrevendo as experiências realizadas na comprovação da existência de ondas eletromagnéticas e a teoria de Maxwell sobre a propagação da luz. A explicação do fenômeno é feita a partir da experiência, tudo de maneira bem detalhada. Logo em seguida são analisados os aspectos que não podem ser explicados em termos da teoria ondulatória clássica da luz. Para explicar o efeito, Einstein colocou em questão a teoria clássica da luz e propôs uma nova teoria, onde o efeito fotoelétrico testaria qual teoria estava correta. A teoria quântica de Einstein propôs que a energia era quantizada em pacotes concentrados de energia, o que mais tarde passou a ser chamado de fótons. Ficava assim explicado o efeito fotoelétrico e com ele confirmada a teoria quântica proposta por Einstein.

Quando estudamos o mesmo efeito no curso de Mecânica Quântica averiguamos que o tema abordado simplesmente relata o mesmo estudo histórico estudado no curso de Física Moderna, sendo o efeito citado junto com o experimento, mas não sendo explicados os procedimentos sugeridos na realização da experiência. Os aspectos conflitantes entre o efeito fotoelétrico e a teoria ondulatória da luz também são citados, e em seguida surge a teoria quântica de Einstein, considerando a radiação como constituída de pacotes concentrados de energia uma coleção de quanta de energia.

Como ocorreu com a radiação de corpo negro, o efeito fotoelétrico num curso de Física Moderna é explicado e exemplificado e tem-se um caminho a ser percorrido pelo estudante até se chegar à teoria quântica proposta por Einstein, que é explicada no curso. Isso não acontece quando estudamos o fenômeno na disciplina Mecânica Quântica. O tema não é explicado para um estudante que esteja iniciando os seus estudos em Mecânica Quântica.

No curso de Física Moderna a confirmação da natureza corpuscular da radiação é explicada através da experiência desenvolvida por Compton utilizando um feixe de raios $\mathrm{X}$ de comprimento de onda $\lambda$ incidindo sobre um alvo de grafite (efeito Compton). O fenômeno é explicado com o espalhamento do feixe incidente, que depois de espalhado apresenta um comprimento de onda diferente, o que não está de acordo com o modelo clássico para radiação. O efeito é explicado pensandose que o feixe é constituído de fótons que após a colisão com elétrons dos átomos de grafite, são espalhados com uma energia menor, devido à colisão, apresentando por isso um comprimento de onda diferente do feixe incidente. O efeito é explicado qualitativamente e quantitativamente, e além desse efeito complementa-se o assunto explicando-se o espalhamento Thomson.

Estudando o mesmo efeito em um livro adotado no curso de Mecânica Quântica, observamos que o mesmo explica o fenômeno de forma mais sucinta preocupandose bastante com o aspecto quantitativo, mas não deixando que o efeito fique mal interpretado qualitativamente. Aqui o espalhamento Thomson não chega nem a ser citado. O que se faz é uma pequena referência entre a colisão do fóton com um elétron e a colisão do fóton com o átomo, não explicando a diferença entre as duas colisões.

Nos dois cursos como pudemos ver o efeito é explicado, o que existe de diferente entre os dois é a forma como cada um se dedica ao tema abordado, um se preocupa mais com a compreensão do problema e das implicações que surgem decorrentes do mesmo, esclare- 
cendo a importância do efeito para a teoria quântica da radiação não se esquecendo da comprovação matemática do fenômeno. O outro prioriza o processo matemático que explica o efeito simplificando a compreensão do mesmo.

Além do efeito fotoelétrico e do efeito Compton outros exemplos que comprovam a natureza corpuscular da radiação são apresentados em um curso normal de Física Moderna, como, por exemplo, a produção de raios $\mathrm{X}$ e a produção e a aniquilação de pares, exemplos que no curso de Mecânica Quântica necessariamente são omitidos, não por não serem importantes, mas devido ao fato de que sua omissão não prejudica a comprovação das propriedades corpusculares da radiação.

Depois de analisar as propriedades corpusculares da radiação contidas em cada um dos cursos, analisamos as propriedades ondulatórias das partículas. Verificamos que no curso de Física Moderna existe uma certa preocupação na localização histórica do fenômeno, começando com a hipótese de Louis de Broglie, de que a uma partícula material tem associada uma onda de matéria que governa seu movimento, comprovada posteriormente com a experiência de Davisson-Germer e a de Thomson, que são explicadas no curso. O tema é desenvolvido partindo-se dessa hipótese de Louis de Broglie da existência de ondas de matéria, chegando até a interpretação feita por Born da dualidade ondapartícula da matéria, que no texto é comparada com a dualidade onda-partícula da radiação interpretada por Einstein. Dessa analogia surgem as evidências que levam ao princípio da incerteza de Heisenberg e suas conseqüências, o que possibilitou o estudo das propriedades das ondas de matéria.

Em linhas bem gerais o curso de Mecânica Quântica trata a interpretação da dualidade da matéria, preocupando-se bastante com a interpretação matemática do princípio de incerteza. Nesse curso as relações de incertezas são encontradas com a utilização das integrais de Fourier, sendo também expostos a interpretação, o enunciado e as conseqüências do princípio da incerteza de Heisenberg.

Confrontando a forma como nos dois cursos é abordado o tema, notamos que o curso de Física Moderna se preocupa rigorosamente com a interpretação da dualidade da matéria, mas que necessariamente, por não ter esse propósito, não comprova matematicamente o princípio da incerteza utilizando as integrais de Fourier. Devemos salientar também, por ser importante, que nos dois cursos o princípio da incerteza de Heisenberg, e suas relações, surgem como conseqüência do postulado de de Broglie.

Por muito tempo se procurou um modelo que pudesse representar o átomo, desvendando a natureza do mesmo. No curso de Física Moderna o modelo para o átomo recebe uma especial atenção, começando pelo modelo de Thomson (uma esfera de cargas positivas entremeada por elétrons) estudando-se as propriedades do modelo e os fatos que levaram o modelo ao fracasso, as experiências de Geiger e Marsden sobre o espalhamento de partículas alfa através de lâminas finas, determinando a necessidade de outro modelo que conseguisse explicar os fatos que foram observados. Dessas observações, feitas por Rutherford, constrói-se o modelo de Rutherford, onde as cargas positivas do átomo, e conseqüentemente toda sua massa são supostas concentradas no centro chamado núcleo, e os elétrons girando ao redor desse núcleo em órbitas circulares. Segundo esse modelo o elétron acelerado emitia radiação diminuindo sua energia tendendo a cair em direção ao núcleo, o que não acontece. Para explicar o fato Bohr postulou que o elétron em uma órbita estacionária não emite radiação eletromagnética, só emite ou absorve radiação quando realiza transições entre estados de maior ou menor energia, postulando também que o momento angular do elétron é quantizado (apenas determinadas órbitas são permitidas!). Neste curso são estudados ainda os estados de energia do átomo, o princípio da correspondência, a interpretação das regras de quantização de Wilson-Sommerfeld e o modelo atômico com órbitas elípticas de Sommerfeld.

Os modelos que representam a natureza do átomo, não recebem um tratamento especial quando estudados em um curso de Mecânica Quântica. O modelo de Thomson nesse curso é citado e explicado em poucas linhas, junto com a experiência de Geiger e Marsden e logo em seguida comenta-se o modelo de Rutherford chegando até o ponto onde esse modelo não consegue explicar por que o elétron não cai em direção ao núcleo, como o modelo sugeria. Para esclarecer a questão o livro adotado nesse curso já elucida o problema com os postulados de Bohr, ou seja, o modelo de Bohr para o átomo. Aqui os níveis de energia do átomo são estudados, no entanto, a interpretação das regras de quantização é resumida numa proposição bem geral, de Wilson-Sommerfeld, mencionada no texto. $\mathrm{O}$ princípio de correspondência é enunciado e o modelo de Sommerfeld não é nem citado no livro do curso.

Comparando o que foi exposto sobre os modelos atômicos contidos nos dois cursos, podemos dizer que em Física Moderna o tema é bastante discutido fornecendo-se uma base sólida sobre o assunto (um capítulo inteiro do livro utilizado nesse curso é para o estudo dos modelos) e que no curso de Mecânica Quântica o tema é abordado de maneira diferente, como uma revisão. No curso de Mecânica Quântica apenas uma seção de um capítulo é dedicada para expor este tópico.

No curso de Física Moderna as leis do movimento ondulatório de uma partícula são tratadas com a equação de onda encontrada por Schrödinger. Nesse curso primeiro se discute o papel da teoria e sua relação com o postulado de De Broglie, continuando com os argumentos plausíveis para se chegar à equação de Schrödinger: a necessidade de ser uma equação de onda 
diferencial, de ser linear e consistente com as equações de Newton e com os postulados de De Broglie-Einstein. Em seguida analisa-se a interpretação de Born para funções de onda, onde se estabelece que as previsões em Mecânica Quântica são probabilísticas (o que se determina são os valores esperados!). Nesse capítulo os operadores, as equações para operadores e a fórmula geral para o cálculo de valores esperados são estudados. O método de separação de variáveis é utilizado para obter a equação de Schrödinger independente do tempo, que é discutida junto com os argumento para a equação. Estudam-se ainda as autofunções, os autovalores, as propriedades necessárias às autofunções e a quantização da energia na teoria de Schrödinger.

A equação de onda de Schrödinger também é estuda na disciplina Mecânica Quântica. O tema é abordado iniciando-se pela equação diferencial, relacionando a equação com o postulado de de Broglie. Chega-se à conclusão de que as previsões em Mecânica Quântica são probabilísticas. É estudada a interpretação de Max Born para a densidade de probabilidade. Os operadores posição, momento, e energia são interpretados. A fórmula geral para o cálculo de valores esperados é fornecida. A equação de Schrödinger independente do tempo é encontrada com a utilização do método de separação de variáveis. Os argumentos para a equação são rapidamente mencionados. Os autovalores, as autofunções e suas propriedades são estudados. A quantização da energia também é citada.

Avaliando a forma como é abordado o assunto nos dois cursos, notamos que a equação de Schrödinger recebe um tratamento semelhante em ambos. A grande diferença é que no curso de Mecânica Quântica o assunto é tratado de uma forma mais ampla e com um maior refino matemático, falando um pouco mais nos operadores. No curso de Mecânica Quântica estudamse a corrente de probabilidade, fluxo, tema que não chega a ser mencionado no curso de Física Moderna. A teoria de Schrödinger é analisada em apenas um capítulo no curso de Física Moderna e em dois no de Mecânica Quântica.

Analisamos também as aplicações da equação de Schrödinger independente do tempo contidas no curso de Física Moderna. Primeiro resolve-se a equação de Schrödinger independente do tempo para um potencial nulo, encontrando as soluções e interpretando as autofunções e as funções de onda. Depois é estudado o potencial degrau com a energia da partícula menor que a altura do degrau, sendo estudadas a autofunção geral para o degrau, limitações, condições de continuidade no degrau, o coeficiente de reflexão, penetração sob o degrau e o limite clássico. Logo em seguida é estudado o potencial degrau com energia maior que a altura do degrau. Em continuidade é analisada a barreira de potencial, estudando-se a densidade de probabilidade, o coeficiente de transmissão através da barreira, o efeito túnel, o espalhamento elétron-átomo, o efeito Ramsauer e ressonância de forma, citada no capítulo. Por fim a barreira é comparada com o degrau sendo ainda estudados exemplos de penetração de barreiras por partículas. $\mathrm{O}$ poço de potencial quadrado com barreiras finitas também é estudado. Depois é introduzido o poço de potencial quadrado infinito. Sua solução analítica é encontrada bem como os autovalores, a energia do estado fundamental não nula e sua a relação com o princípio da incerteza, as autofunções, a aplicação direta da relação de De Broglie, o elétron ligado ao núcleo e o limite clássico. Em seguida é estudado o potencial do oscilador harmônico simples, sendo encontrados para as pequenas vibrações, os autovalores, a energia do estado fundamental e as autofunções. A questão da paridade também é analisada aqui.

As aplicações da equação de Schrödinger independente do tempo no curso de Mecânica Quântica, cujo capítulo é intitulado como potenciais unidimensionais, é analisado no texto porque são problemas simples que ilustram efeitos não-clássicos. Nesse curso a primeira aplicação analisada é o potencial degrau, tanto o com energia maior que o degrau quanto o com energia menor que o degrau. Depois do potencial degrau estuda-se o poço de potencial: o com barreiras finitas é interpretado e o com barreiras infinitas é mencionado. Em seguida estuda-se a barreira de potencial, analisando o coeficiente de transmissão através da técnica de aproximação de Wentzel-Kramers-Brillouin. O fenômeno de tunelamento é explicado e exemplos são discutidos no capítulo. O modelo unidimensional da molécula de amônia é exemplificado e analisado. O modelo de Kronig-Penney é explicado no curso de Mecânica Quântica, o qual é importante na teoria dos metais, dos não-condutores e dos semicondutores. De maneira análoga ao curso de Física Moderna por último é estudado o oscilador harmônico simples.

Confrontando o tema abordado nos dois cursos, o de Física Moderna e o de Mecânica Quântica, verificamos que em ambos o assunto é tratado de maneira similar, apresentando diferenças principalmente na forma como se colocam os exemplos. Um detalhe importante a ser citado, comentado anteriormente, é o fato de que no curso de Mecânica Quântica a discussão dos exemplos, em boa parte, é feita de maneira matematicamente mais refinada, o que evidência a necessidade de que o estudante do curso possua uma maior habilidade matemática que pode ser conseguida através de cursos de Física Matemática. Um exemplo disso foi a utilização da técnica de aproximação de WentzelKramers-Brillouin e em alguns casos da função delta de Dirac. No curso de Física Moderna o modelo de Kronig-Penney não é explicado e nem citado. No curso de Mecânica Quântica o efeito túnel e a ressonância de forma não são mencionados e o efeito Ramsauer é citado no poço de potencial e não na barreira de potencial.

Átomos de um elétron é um tema considerado muito importante para o estudo da mecânica quântica, pois 
é o caso mais simples onde a equação de Schrödinger em três dimensões é resolvida. A partir daí é que se faz a generalização para átomos de muitos elétrons. No curso de Física Moderna primeiro analisa-se a importância de átomos de um elétron e a vantagem de se trabalhar com o elétron de massa reduzida. Em seguida desenvolve-se a equação de Schrödinger em três dimensões sendo encontrada a equação independente do tempo. O próximo passo é transformar a equação independente do tempo de coordenadas retangulares para coordenadas esféricas, separando a equação independente do tempo em três equações: uma em $r$, uma em $\theta$ e outra em $\phi$, encontrando as suas soluções. Os quatro números quânticos são estudados. Estuda-se ainda os autovalores, a degenerescência, as autofunções, a densidade de probabilidade, o momento angular orbital.

O átomo de hidrogênio no curso de Mecânica Quântica também é resolvido, mostrando a importância do mesmo para o estudo da Mecânica Quântica. Nesse curso a forma como vem desenvolvido o tema é diferente do curso de Física Moderna. Aqui antes de resolvermos o átomo de hidrogênio, estudamos a estrutura geral da mecânica ondulatória, o método de operadores em mecânica quântica, o sistema de $N$ partículas, a equação de Schrödinger em três dimensões, o momento angular e resolvemos a equação radial. Depois desse estudo resolvemos então o átomo de hidrogênio, cuja solução é encontrada pelo mesmo método utilizado no curso de Física Moderna.

No curso de Física Moderna estudamos o momento de dipolo magnético, spin e taxas de transição. Ao estudarmos o momento de dipolo magnético orbital começamos analisando o momento de dipolo magnético e o momento angular de um elétron em órbita, o chamado magnéton de Bohr, o fator orbital, a precessão de Larmor, o dipolo magnético num campo magnético uniforme, e os efeitos de um campo magnético não uniforme. Depois analisamos a experiência de SternGerlach e estudamos o spin do elétron. É vista a exemplificação do dispositivo experimental, a quantização espacial, o acordo e desacordo qualitativo com as previsões de Schrödinger, a experiência de PhilippsTaylor, o spin, números quânticos, o momento angular de spin, momento de dipolo magnético, o efeito Zeeman, o spin e a estrutura fina, o caráter não clássico do spin, e a teoria relativística de Dirac. Em seguida passa-se para a interação spin-órbita, onde são estudados o campo magnético interno num átomo de um elétron, o momento de dipolo magnético de spin e sua energia orientacional, a precessão de Thomas, e a energia de interação spin-órbita. Após isto é analisado o momento angular total, o acoplamento entre o momento angular orbital e de spin, o comportamento do momento angular total e as condições satisfeitas pelos números quânticos. Termina-se o assunto estudando a relação energia de interação spin-órbita e os níveis de energia do hidrogênio, as taxas de transição e regras de seleção.

Em um curso de Mecânica Quântica a visão do conteúdo é outra, para ser mais claro, o curso dedicase a um estudo mais aprofundado do assunto, exigindo que o estudante possua conhecimento em Física Matemática. O tema é abordado nesse curso tratando especialmente da interação de elétrons com o campo eletromagnético, depois se estuda os operadores, matrizes e spin. É estudado também a adição de momentos angulares. Ao estudar a interação de elétrons com o campo eletromagnético, são vistos o efeito Zeeman normal e o efeito Bohm-Aharanov, o primeiro citado no curso de Física Moderna, e o outro não é citado no mesmo curso. Os operadores e as matrizes não são estudados no curso de Física Moderna, o spin é estudado, porém de uma forma diferente, pois no curso de Mecânica Quântica utilizam-se matrizes em sua descrição, o que não acontece no curso de Física Moderna. Verificamos que ao se estudar a interação de elétrons com o campo eletromagnético, operadores, matrizes e spin, encontramos um ponto onde os dois cursos diferenciam-se completamente na abordagem do conteúdo.

Ainda no curso de Física Moderna estudamos os átomos multieletrônicos a partir do estudo das partículas idênticas e suas propriedades físicas, chegando até o princípio de exclusão de Pauli, enunciado fraco e o enunciado forte do princípio, determinantes de Slater, férmions, bósons e a relação entre spin e simetria. Estudamos a autofunção singleto e autofunção simétrica. Logo após é introduzido um estudo da tabela periódica. O curso de Física Moderna geralmente termina com esse tema.

No curso de Mecânica Quântica o tema também é estudado. São vistos o princípio de exclusão de $\mathrm{Pa}-$ uli, determinantes, férmions, bósons, simetria, as autofunções singleto e tripleto e a tabela periódica. O assunto nesse curso é aprofundado e acrescido de novos conteúdos. Um exemplo que podemos citar é a teoria das perturbações independentes do tempo, aplicação do campo elétrico no átomo de hidrogênio, que não são estudados no curso de Física Moderna. Ainda no curso de Mecânica Quântica são estudados outros conteúdos que não são vistos no curso de Física Moderna, como por exemplo o átomo de hidrogênio real e o átomo de hélio.

\section{Comentários Finais}

Verificamos neste trabalho que a disciplina de Física Moderna para Licenciatura é muito importante, visto que praticamente quase todos os fenômenos modernos verificados no século XX só podem ser explicados com a Mecânica Quântica. Torna-se então necessário ao professor de ensino médio o conhecimento desta disciplina, visto que estamos rodeados por tais fenômenos 
quânticos em nosso cotidiano. Notamos também que em algumas universidades a Física Moderna já faz parte do programa de Física exigido no vestibular. Outra importância do curso de Física Moderna é que ele é uma introdução necessária para os estudantes de Licenciatura em Física que desejam prosseguir seus estudos fazendo o mestrado em Física.

Quanto à comparação entre o curso de Física Moderna e o de Mecânica Quântica, verificamos que o conteúdo dos dois cursos é praticamente o mesmo. A maior discrepância entre as duas disciplinas é que o curso de Mecânica Quântica prioriza o processo matemático (aspecto "quantitativo") utilizando métodos refinados de Física Matemática, não havendo, entretanto nenhuma perda em termos de contexto (aspecto "qualitativo") aos alunos de Licenciatura em Física no que se refere ao estudo da Física Quântica. Podemos afirmar que o curso de Física Moderna é uma introdução à Mecânica Quântica, e o curso de Mecânica Quântica é um estudo mais aprofundado do assunto. Assim o curso de Física Moderna deve ser visto como pré-requisito para o curso de Mecânica Quântica.
Uma questão levantada por alunos do curso de Licenciatura em Física, os que desejam prosseguir os seus estudos em Física, é por que a disciplina Mecânica Quântica não faz parte da grade curricular do curso? Primeiramente, como já foi dito, para o aluno que vai se dedicar ao ensino de física no ensino médio o curso de Física Moderna é suficiente para o exercício de sua profissão, em segundo acrescentar no curso de Licenciatura em Física a disciplina Mecânica Quântica acarretaria mudanças desnecessárias na grade curricular do curso.

\section{References}

[1] J. L. Lopes, A estrutura quântica da matéria, ed. UFRJ, Rio de Janeiro (1993).

[2] S. Gasiorowicz, Física quântica, ed. Guanabara Dois, Rio de Janeiro (1979).

[3] R. Eisberg, R. Resnick, Física quântica: átomos moléculas, sólidos, núcleos e partículas, ed. Campus, Rio de Janeiro (1988). 